\title{
Modeling an induction motor based on the equations of a generalized electric machine, taking into account the saturation of the magnetic circuit
}

\author{
Yuri Mikhailovich Frolov ${ }^{1}$, Valery Petrovich Shelyakin ${ }^{1}$, Nikolay Vasilievich Sitnikov ${ }^{1}$, Sergey Aleksandrovich \\ Goremykin ${ }^{1}$, and Dmitry Aleksandrovich Tonn ${ }^{1}$ \\ ${ }^{1}$ Voronezh State Technical University
}

\begin{abstract}
Writing the differential equations of an asynchronous electric motor on the basis of a generalized electric machine in different coordinate axes can not only reduce the number of model equations, but also introduce various factors in the mathematical description that increase the modeling accuracy. It is proposed to write the equations of the mathematical model of an induction motor taking into account the saturation of the magnetic circuit and solve them using the Matlab application package. The proposed approach improves the accuracy of calculating the mechanical characteristics of an asynchronous electric drive.
\end{abstract}

\section{Introduction}

An important step in the synthesis and analysis of electric drive systems is the development of a model of an electric machine that meets the challenges of vector control [1-6].

In most cases, electromechanical energy converters are made in multiphase design. This fact complicates the mathematical apparatus that describes the transient processes in such converters. This is due to an increase in the number of equations of the mathematical model of the experimented electric machine. Therefore, as a rule, they strive to make an equivalent transition from a real machine to a generalized double-phased [7, 8]. The generalized machine in the mathematical description is characterized by the following generally accepted assumptions: "the phase windings are symmetrical, the air gap is uniform, there are no losses in steel and tooth phenomena, the magnetizing circuit is linear, the magnetizing force of each winding and the magnetic induction in the air gap are sinusoidal around the circumference of the stator (rotor), rotor parameters are reduced to the stator circuit "[7-9]. The equations of the mathematical model of a generalized machine can be written in different forms depending on the coordinate systems used. The most commonly used coordinate systems are: $u, v ; \alpha, \beta ; d, q[7,8]$.

\section{Methods}

We write the equations of the generalized machine in the coordinate system $u, v$ rotating synchronously with the speed of the electromagnetic field:

$$
\begin{aligned}
& U_{1 v}=\left(R_{1}+p \cdot L_{1}\right) \cdot i_{1 v}+p \cdot L_{12} \cdot i_{2 v}- \\
& -L_{1} \cdot \omega_{k} \cdot i_{1 u}-L_{12} \cdot \omega_{k} \cdot i_{2 u}, \\
& U_{1 u}=\left(R_{1}+p \cdot L_{1}\right) \cdot i_{1 u}+p \cdot L_{12} \cdot i_{2 u}- \\
& -L_{1} \cdot \omega_{k} \cdot i_{1 v}-L_{12} \cdot \omega_{k} \cdot i_{2 v}, \\
& U_{2 u}=\left(R_{2}+p \cdot L_{2}\right) \cdot i_{2 u}+p \cdot L_{12} \cdot i_{1 u}- \\
& -L_{12} \cdot\left(\omega_{k}-\omega_{R}\right) \cdot i_{1 v}-L_{2} \cdot\left(\omega_{k}-\omega_{R}\right) \cdot i_{2 v}, \\
& U_{2 v}=\left(R_{2}+p \cdot L_{2}\right) \cdot i_{2 v}+p \cdot L_{12} \cdot i_{1 v}+ \\
& +L_{12} \cdot\left(\omega_{k}-\omega_{R}\right) \cdot i_{1 u}+L_{2} \cdot\left(\omega_{k}-\omega_{R}\right) \cdot i_{2 u}, \\
& M=P_{n} \cdot L_{12} \cdot\left(i_{1 v} \cdot i_{2 u}-i_{1 u} \cdot i_{2 v}\right) .
\end{aligned}
$$

where $R_{1}, R_{2}$ - active resistance of stator and rotor windings, respectively;

$L_{1}, L_{2}$ - the total inductances of the stator and rotor windings, respectively;

$L_{12}$ - mutual inductance of stator and rotor windings;

$\omega_{k}$ - angular velocity of rotation of the coordinate axes;

$\omega_{R}=P_{n} \cdot \omega_{\text {rotor }}-$ angular velocity of the rotor of the machine in electrical degrees;

$p$ - differentiation operator;

$P_{n}$ - number of pole pairs.

Equations of a generalized electric machine in a coordinate system $u, v$ - most common. Transition from coordinates $v, u$ to a fixed coordinate system $\alpha, \beta$ occurs by direct coordinate transformation at $\omega_{k}=0$.

$$
\begin{aligned}
& U_{l \alpha}=U_{1 \text { max }} \cdot \cos \left(\omega_{0 R} \cdot t\right), \\
& U_{1 \beta}=U_{\text {Imax }} \cdot \sin \left(\omega_{0 R} \cdot t\right),
\end{aligned}
$$


where $U_{1 \max }=\sqrt{3} \cdot U_{1 \text { phasevoltage }}$.

Transition from coordinate system $u, v$ to the coordinate system $d, q$ rotating at the speed of the rotor, if we assume that $\omega_{k}=\omega_{R}$. From here, the system of phase voltages applied to the windings will be equal:

$$
\begin{aligned}
& U_{l d}=U_{I \max } \cdot \cos ^{2}\left(\omega_{0 R} \cdot t\right)+ \\
& +U_{I \max } \cdot \sin ^{2}\left(\omega_{0 R} \cdot t\right)=U_{1 \max }, \\
& U_{l q}=-U_{I \max } \cdot \sin \left(\omega_{0 R} \cdot t\right) \cdot \sin \left(\omega_{O R} \cdot t\right)+ \\
& +U_{I \max } \cdot \sin \left(\omega_{O R} \cdot t\right) \cdot \cos \left(\omega_{O R} \cdot t\right)=0 .
\end{aligned}
$$

The system of equations (1) corresponds to the structure of the electromechanical energy converter (EMF), shown in figure 1. This structure was compiled in the Simulink application of the Matlab application package [10-12]. Here, the voltages $U_{1 u}, U_{1 v}, U_{2 u}, U_{2 v}$ are transformed control actions connecting the engine with the control system in the axes $\mathrm{x}, \mathrm{y}$.

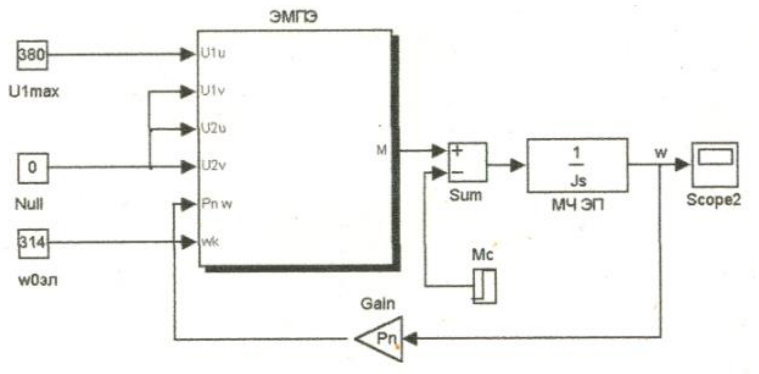

Fig. 1. Structural diagram of EMF and the mechanical part of ES.

Transients in electromechanical converters are classified as follows: during start-up, braking, reverse, restart, and also when the load changes [13-16]. To obtain information about the features of transient processes in an electric machine, reliable data on the parameters and independent variables of the model are needed.

In most cases, the nature of transient processes in EMF affects the configuration of the drive as a whole. Namely: overall dimensions, permissible electromagnetic loads, operating modes, including the features of starting and braking these systems.

\section{Results}

Figures 2, 3 below show the laws of change in the angular velocity of rotor 1 and electromagnetic moment 2 in time for an induction motor 4A80B6Y3 of power $1,1 \mathrm{~kW}, P_{n}=3, U=220 / 380$. So, figure 2 illustrates the idle mode at the moment of inertia equal to the intrinsic moment of inertia of the rotating parts of the $\operatorname{EMF}(J=$ $J_{\mathrm{M}}$ ).

Figure 3 characterizes the transient processes when the load on the shaft is equal to the nominal and at a double value of the moment of inertia $\left(J=2 J_{M}\right)$.

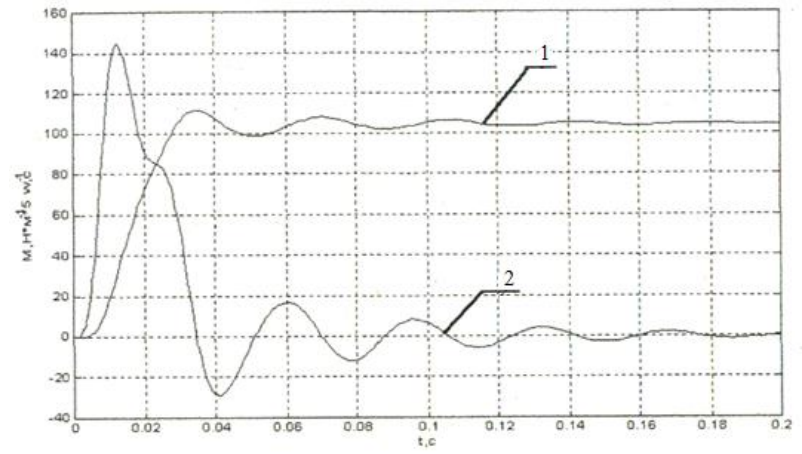

Fig. 2. Transient characteristics at a static moment equal to $M_{C}=0$ and moment of inertia $J=J_{\mathrm{M}}$.

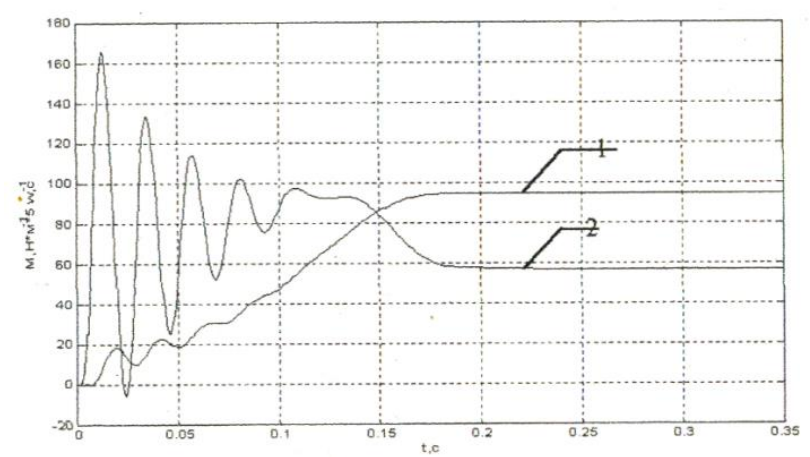

Fig. 3. Transient characteristics at a static moment equal to the rated $M_{C}=M_{N}$ and moment of inertia $J=2 J_{\mathrm{M}}$.

In order to optimize the EMF by mass and size indicators, the "operating point" is selected on a nonlinear section of the magnetization characteristic. When changing the parameters of the supply network, the degree of saturation of the magnetic circuit of the machine changes. This leads to the fact that the intrinsic and mutual inductances between the windings change, therefore, it is necessary to take into account changes in the inductive parameters of the electric machine over time $[17,18]$. Variation of these parameters, in turn, will lead to a variety of EMF characteristics, both in steady state and in transient modes.

When starting the above-considered asynchronous electric motor due to the presence of alternating components in the amplitudes of the stator and rotor currents, and, accordingly, the magnetization current, the magnetic circuit of an electric machine can enter a deep saturation state. The inductance of the magnetization and scattering circuit of the windings of a saturated machine significantly differ from the inductances of the nominal mode, and with increasing saturation the inductance decreases. Within the limits of calculation error, it can be assumed that the magnetic flux of scattering closes through the air, therefore, we can assume that the inductive parameters of the stator and rotor will not depend on saturation.

The graphs of changes in inductance versus magnetization current will be obtained from the relationships shown in figure 4.

From curve 2, for each value of the magnetization current, we find the value of the open-circuit emf, which is equal (in relative units) to the value of flux linkage in the air gap. Knowing the magnitude of flux linkage 
corresponding to a certain current value $I_{\mu}$, we find the reciprocal of the inductance (in relative units) $l / L_{\mu}$ along curve 1 .

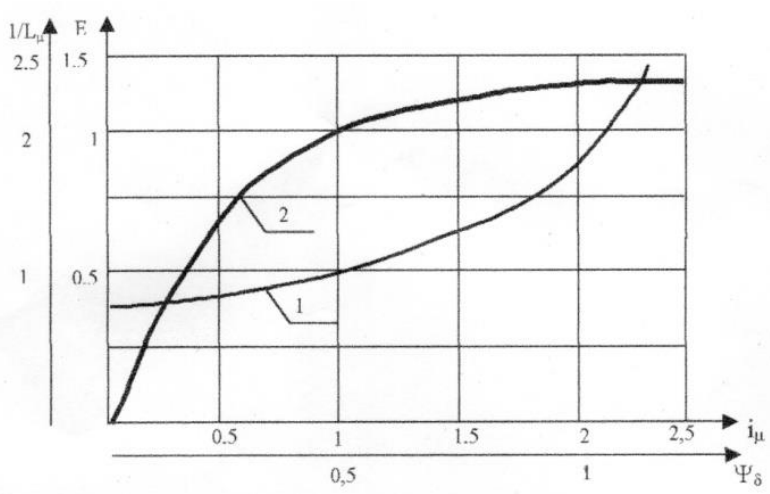

Fig. 4. Universal BP magnetization curves.

Approximate relationship $L_{\mu}=f(I \mu)$ is shown in figure 5 .

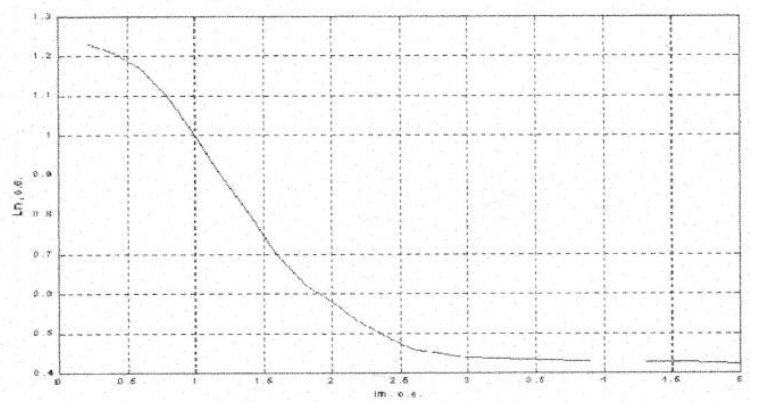

Fig. 5. Relationship $L_{\mu}=f(I \mu)$.

The magnetization current can be determined by adding the projections of the stator and rotor currents on the axis $u$,v. As $I_{\mu}=I_{1}+I_{2}$, then from the vector diagram shown in figure 6 , it can be seen that:

$$
\begin{aligned}
& I_{\mu u}=I_{1 u}+I_{2 v} \\
& I_{\mu v}=I_{1 v}+I_{2 v}
\end{aligned}
$$

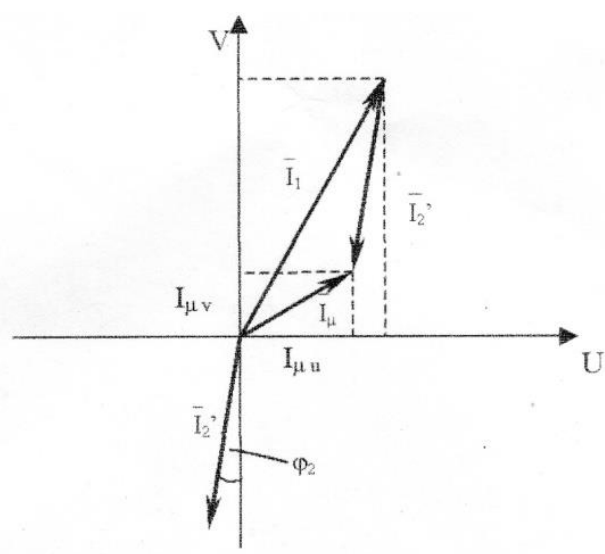

Fig. 6. Vector diagram of currents in axles U and V.
The magnitude of the magnetization current from equation (5), (6) is determined by the following formula:

$$
I_{\mu}=\sqrt{\left(I_{1 u}-I_{2 u}\right)^{2}+\left(I_{1 v}-I_{2 v}\right)^{2}}
$$

The equations of an asynchronous machine, taking into account the saturation of the magnetic circuit, are written as follows

$$
\begin{aligned}
& U_{1 v}=\left(R_{1}+p \cdot L_{1}(I \mu)\right) \cdot i_{1 v}+p \cdot L_{12}(I \mu) \cdot i_{2 v}- \\
& -L_{1}(I \mu) \cdot \omega_{k} \cdot i_{1 u}-L_{12}(I \mu) \cdot \omega_{k} \cdot i_{2 u}, \\
& U_{1 u}=\left(R_{1}+p \cdot L_{1}(I \mu)\right) \cdot i_{1 u}+p \cdot L_{12}(I \mu) \cdot i_{2 u}- \\
& -L_{1}(I \mu) \cdot \omega_{k} \cdot i_{1 v}-L_{12}(I \mu) \cdot \omega_{k} \cdot i_{2 v}, \\
& U_{2 u}=\left(R_{2}+p \cdot L_{2}(I \mu)\right) \cdot i_{2 u}+p \cdot L_{12}(I \mu) \cdot i_{1 u}- \\
& -L_{12}(I \mu) \cdot\left(\omega_{k}-\omega_{R}\right) \cdot i_{1 v}-L_{2}(I \mu) \cdot\left(\omega_{k}-\omega_{R}\right) \cdot i_{2 v}, \\
& U_{2 v}=\left(R_{2}+p \cdot L_{2}(I \mu)\right) \cdot i_{2 v}+p \cdot L_{12}(I \mu) \cdot i_{1 v}+ \\
& +L_{12}(I \mu) \cdot\left(\omega_{k}-\omega_{R}\right) \cdot i_{1 u}+L_{2}(I \mu) \cdot\left(\omega_{k}-\omega_{R}\right) \cdot i_{2 u}, \\
& M=P_{n} \cdot L_{12}(I \mu) \cdot\left(i_{1 v} \cdot i_{2 u}-i_{1 u} \cdot i_{2 v}\right) .
\end{aligned}
$$

Graphs of transients of the magnetization current (curve 1) and magnetization inductance (curve 2) when starting an induction motor $4 \mathrm{~A} 80 \mathrm{~B} 6 \mathrm{Y} 3$ of power $1,1 \mathrm{~kW}$, $P_{n}=3, U=220 / 380$, when the load on the shaft is zero is shown in figure 7. Transition charts speed (curve 1) and electromagnetic moment (curve 2), under similar experimental conditions, are shown in figure 8 .

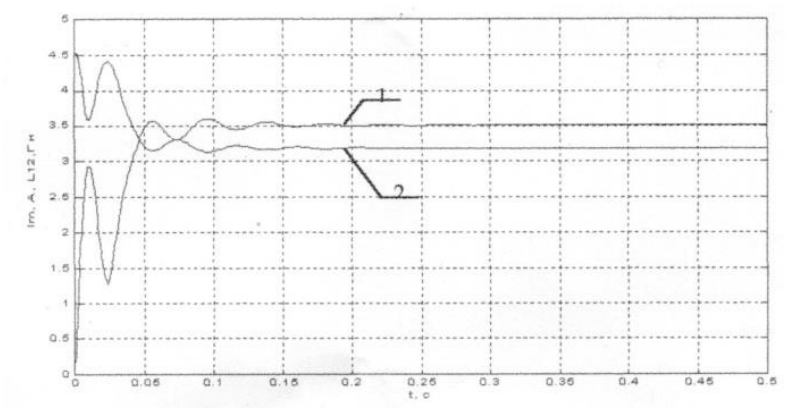

Fig. 7. Graphs of transients of the magnetization current and magnetization inductance at start-up.

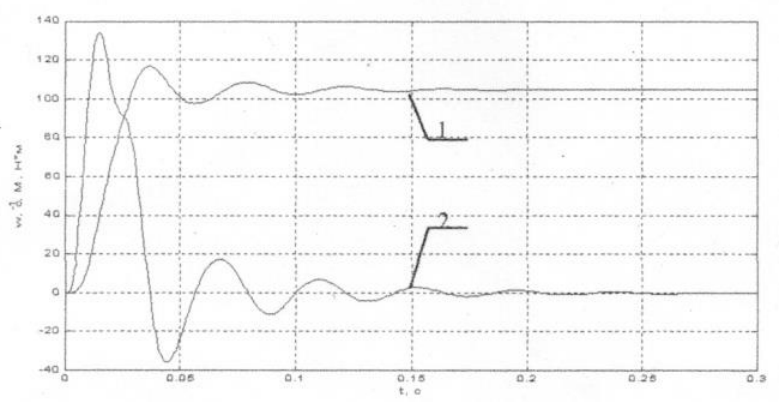

Fig. 8. Graphs of transient processes of speed and electromagnetic moment at start-up to idle.

Graphs of transients of the magnetization current (curve 1) and magnetization inductance (curve 2) when starting an induction motor with a load on the shaft equal to the nominal value is shown in figure 9. Graphs of transients of speed (curve 1) and electromagnetic 
moment (curve 2), under similar conditions conducting experiments are shown in figure 10.

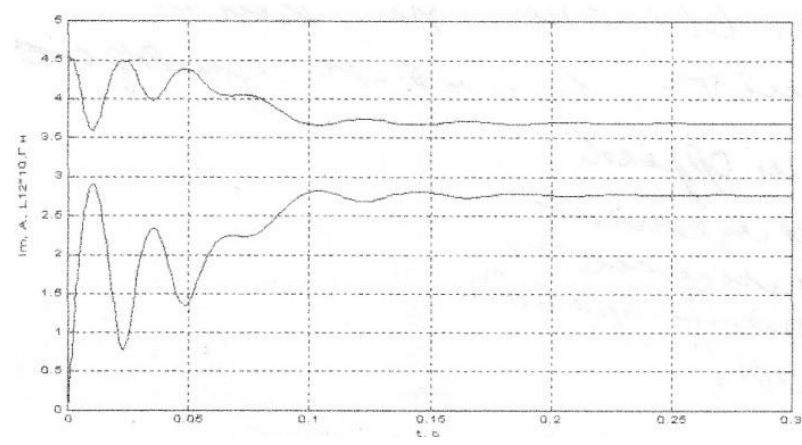

Fig. 9. Graphs of transients of the current and magnetization inductance at start, loaded with a rated torque, of the motor.

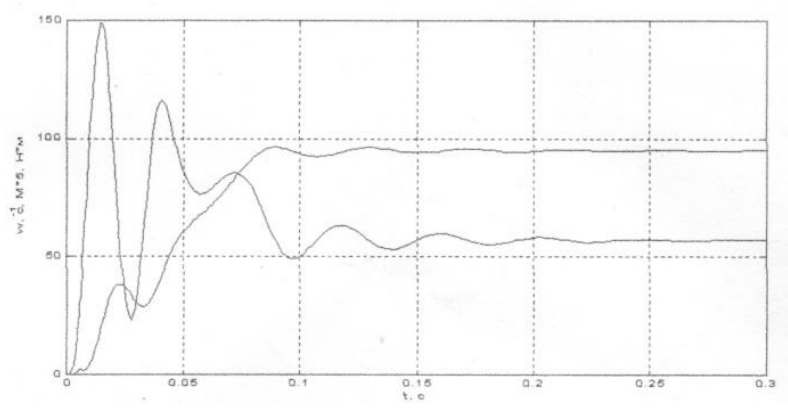

Fig. 10. Graphs of transient processes of speed and electromagnetic moment loaded with rated torque of the engine.

\section{The discussion of the results}

The availability of reliable information about the nature of the transition processes in modern electric drives makes it possible to increase the reliability and accuracy of the operation of critical actuators. For a more accurate mathematical description of transients, various generally accepted assumptions are removed. When trying to take into account an assumption, a differentiated approach is needed. The essence of this approach is that we take into account only those factors that, for the considered group of machines and their operating conditions, have a significant impact on the accuracy of the calculation of the mathematical model. In the framework of this work, the possibility of taking into account such an important factor as the saturation of the magnetic circuit in the calculation of transients in a specific induction motor using modern Matlab application software is shown.

\section{References}

[1] A.E. Kravchik, M.M. Schlaf, V.I. Afonin, et al. A Series Induction Motors: Reference Book (Moscow: Energoizdat, 504, 1982)

[2] V.N. Brodsky, E.S. Ivanov, Drives with frequency - current control (M .: Energy, 168, 1974)

[3] A.V. Ivanov-Smolensky, Electric cars: Textbook for high schools (M.: Energy, 928, 1980)
[4] V.I. Klyuchev, Theory of electric drive: Textbook for universities (M.: Energoatomizdat, 560, 1985)

[5] S.A. Kovchin, Yu.A. Sabinin, Theory of electric drive: Textbook for high schools (St. Petersburg: Energoatomizdat, St. Petersburg branch, 496, 2000)

[6] V.V. Rudakov, I.M. Stolyarov, V.A. Dartau, Asynchronous electric drives with vector control (L.: Energoatomizdat, 136, 1987)

[7] I.P. Kopylov, Mathematical modeling of electrical machines (M.: Higher School, 318, 1994)

[8] G.A. Sipailov, E.V. Kononenko, K.A. Ferrets, Electric machines (special course) (M.: Higher School, 287, 1987)

[9] Yu.M. Frolov, V.A. Trubetskoy, P.Yu. Tarasov, Modeling the system of frequency - current control of an asynchronous electric drive, Automation and robotization of technological processes: Materials of regional scientific and technical. conferences, Voronezh: Publishing House of VSTU, 121-129 (2000)

[10] V.P. Dyakonov, MATLAB 6.5 SP1 / 7 + Simulink 5/6 (Basics of application, Series "Professional Library", M.: SOLON-Press, 800, 2005)

[11] V.G. Potemkin, The system of engineering and scientific calculations in Matlab 5.x: 2 tons (M.: Dialog-MEPhI, 1999)

[12] V.E. Kondrashov, S.B. Korolev, MATLAB as a system for programming scientific and technical calculations (M.: Mir, Institute for Strategic Stability, Minatom of the Russian Federation, 350, 2002)

[13] Yu.A. Sabinin, V.L. Cargo, Frequency regulated asynchronous electric drives (L.: Energoatomizdat, 128, 1985)

[14] O.V. Sledzhanovsky, L.K. Datskovsky, I.S. Kuznetsov, et al., Systems of subordinate regulation of $A C$ electric drives with valve converters (M.: Energoatomizdat, 256, 1983)

[15] D. White, G. Woodson, Electromechanical energy conversion (M.- L.: Energy, 528, 1964)

[16] Yu.M. Frolov, R.A. Kosmatykh, S.A. Vlasov, AC electric drive according to the inverter - HELL system: textbook, allowance (Voronezh: Publishing House of VSTU, 130, 1999)

[17] Yu.M. Frolov, A.V. Romanov, Generalized electric machine in the automated design of electric drives, Modern problems of informatization in engineering and technology: Tr. VI International Open Scientific Conference, Voronezh, 53-54 (2001)

[18] Yu.M. Frolov, P.Yu. Tarasov, A.N. Seleznev, The study of a generalized electric machine, taking into account the saturation of the magnetic circuit, Automation and robotization of technological processes: Materials of regional scientific and technical. conferences, Voronezh: Publishing House of VSTU, 112-119 (2001) 